\title{
Three-body resonance states just below the antiproton and hydrogen dissociation threshold
}

\author{
Takuma Yamashita ${ }^{1, *}$ and Yasushi Kino ${ }^{1, * *}$ \\ ${ }^{1}$ Department of Chemistry, Tohoku University, Sendai 980-8578, Japan
}

\begin{abstract}
We analyze two shallow resonance states below the antiproton hydrogen dissociation threshold with a non-adiabatic three-body calculation. Rearrangement correlation between initial channel and protonium formation channel is explicitly included in the total wavefunction. The lower resonance state is in good agreement with the resonance position and width calculated with the R-matrix theory. The higher resonance state which is newly found is closer to the threshold and much narrower than the former resonance. A polarization effect of the hydrogen atom is found to be indispensable to support the resonance state. The accuracy of the present calculation is evaluated by the extended virial theorem. The resonance states calculated in the present work gives shallower relative energy below the dissociation threshold than the Born-Oppenheimer calculation, suggesting that the electron motion which is ignored in latter calculation would give positive energy because the electron is unbound inside the distance.
\end{abstract}

\section{Introduction}

An antiproton $(\overline{\mathrm{p}})$ scattering off a hydrogen atom has attracted attentions as a fundamental case of the matter-antimatter reaction [1-5] supported by the antiproton beam experiments [6-8]. Recent experimental progress in the strict test for the CPT symmetry using antiprotons and antihydrogen atoms $(\overline{\mathrm{H}})$ is remarkable [9-14]. One of the significant basements for these experiments is the synthesis, confinement and manipulation of cold antiprotons and antihydrogen atoms [15-19]. The cold sources of the antiproton and antihydrogen atom can open a vast new scientific field stimulated by interactions between matter and antimatter. Understanding of the fundamental cold antiproton/antihydrogen reaction with atoms is, therefore, of importance in particle, nuclear and atomic physic and quantum chemistry.

There have been a basic question about formation of particle-antiparticle complex, such as $\overline{\mathrm{H}} \mathrm{H}$ and $\overline{\mathrm{p}} \mathrm{H}$, in particle-antiparticle collision. Theoretical studies of the four-body compound $\overline{\mathrm{H}} \mathrm{H}$ based on the Born-Oppenheimer (BO) approximation [20-22] are pioneering works for this question; however, the $\mathrm{BO}$ approximation involves a fundamental difficulty near the critical distance. When the motion of the antiproton and proton in $\overline{\mathrm{H}} \mathrm{H}$ is separated from that of the positron and electron, a dipole moment of the antiproton-proton system decreases as a decrease of the size of the system. The critical distance $\rho_{0}$ where the antiproton-proton dipole can bind the electron is reported to be $\rho_{0}=0.639$ a.u. $[23,24]$ and for the two leptons,

\footnotetext{
*e-mail: t.ymst@dc.tohoku.ac.jp

**e-mail: y.k@m.tohoku.ac.jp
} 
electron and positron, $\rho_{0}=0.7427$ a.u. $[25,26]$. The BO potential vanishes inside the critical distance; namely, the electron motion is ignored when the distance between the two nuclei is smaller than the critical distance. Since the BO states do not include continuum states of the electron or Ps, they are considered to be bound states in the BO approximation. The interaction between the Pn and $\mathrm{e}^{-} / \mathrm{Ps}$, however, cannot be neglected; the complexes $\overline{\mathrm{pH}}$ and $\overline{\mathrm{H}} \mathrm{H}$ should be treated as resonance states whose decay mode is electron/positronium detachment with $\mathrm{Pn}$ formation, $\mathrm{Pn}+\mathrm{e}^{-} / \mathrm{Ps}$. The $\mathrm{Pn}$ is in the highly excited state having principal quantum number of $n>30$ because a ground state of the Pn is located more than $1 \mathrm{keV}$ lower than the resonance state.

The identification of the near-threshold resonance states of $\overline{\mathrm{p}} \mathrm{H}$ (and its charge-conjugated system $\mathrm{p} \overline{\mathrm{H}})$ and $\overline{\mathrm{H}} \mathrm{H}$ close to the thresholds $\overline{\mathrm{p}}+\mathrm{H}(\mathrm{p}+\overline{\mathrm{H}})$ and $\overline{\mathrm{H}}+\mathrm{H}$ is also a serious concern in the realistic cold scattering [27-31]. A recent theoretical investigation for $\overline{\mathrm{p}} \mathrm{H}$ was shown in a $\mathrm{R}$-matrix scattering calculation [32] of $\mathrm{Pn}+\mathrm{e}^{-}$which reported several resonance states below the $\bar{p}+H$ threshold. A non-adiabatic calculation of $\bar{H} H$ was performed with complex/real scaling method that includes the explicit description of Pn + Ps states [33].

In this work, we perform the non-adiabatic three-body calculation for S-wave resonance states of $\overline{\mathrm{p}} \mathrm{H}$ located just below the $\overline{\mathrm{p}}+\mathrm{H}$ threshold by $\sim 1 \mathrm{meV}$. Since the dominant interaction between the antiproton and hydrogen atom is characterized by the induced dipole moment caused by the electric field of the antiproton that is in proportion to $r^{-4}$ where $r$ is the relative distance between the antiproton and hydrogen atom, the three-body system may have bound (resonance) states below the $\overline{\mathrm{p}}+\mathrm{H}$ dissociation threshold. In order to reveal these resonance states, the complex scaling method (CSM) is used to determine the resonance positions and widths.

Atomic units (a.u.; $m_{\mathrm{e}}=\hbar=e=1$ ) are used throughout this paper, except where mentioned otherwise.

\section{Theory}

The total Hamiltonian for $\overline{\mathrm{p}} \mathrm{H}$ is written as

$$
H=\hat{K}_{\overline{\mathrm{p}}}+\hat{K}_{\mathrm{p}}+\hat{K}_{\mathrm{e}^{-}}-\hat{K}_{\mathrm{CM}}-\frac{1}{r_{\mathrm{pe}^{-}}}-\frac{1}{r_{\overline{\mathrm{pp}}}}+\frac{1}{r_{\overline{\mathrm{p}^{-}}}},
$$

where $\hat{K}_{\mathrm{x}}\left(\mathrm{x}=\left\{\overline{\mathrm{p}}, \mathrm{p}, \mathrm{e}^{-}\right\}\right)$is a kinetic energy operator associated with point vector of particle $\mathrm{x}, \hat{K}_{\mathrm{CM}}$ is the kinetic energy operator for center of mass of the system and $r_{\mathrm{xy}}$ is relative distance between particle $\mathrm{x}$ and $\mathrm{y}$. The proton(antiproton) masses are set to be $m_{\mathrm{p}}\left(m_{\overline{\mathrm{p}}}\right)=$ 1836.15267389 .
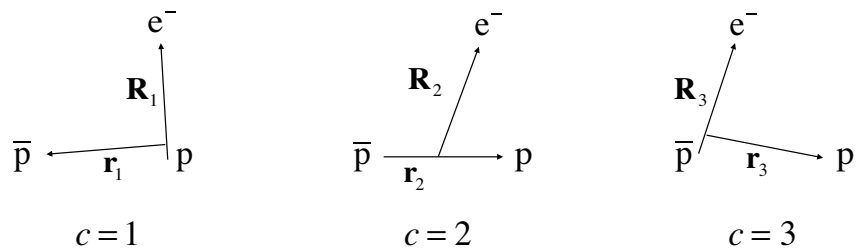

Figure 1. Three sets of Jacobian coordinates for $\overline{\mathrm{p}} \mathrm{H}$. 
In order to describe the all inter-particle correlation of the near-threshold resonance states precisely, we adopt a Gaussian expansion method (GEM) [34]. Recently the GEM approach successfully revealed the near-threshold resonances in positronic atom systems [35-37]. Besides, this approach can be applied to the four-body system $\overline{\mathrm{H}} \mathrm{H}$ in the same theoretical framework [33, 38]. The three-body wavefunction for vibrational quantum number $v$ and total angular momentum $J, \Psi_{v J}$, consists of a summation of channel functions,

$$
\Psi_{v J}=\sum_{c l} \psi_{c l}\left(\mathbf{r}_{c}, \mathbf{R}_{c}\right)
$$

where $\left(\mathbf{r}_{c}, \mathbf{R}_{c}\right)$ are sets of Jacobian coordinates shown in Fig. 1. The first channel $c=1$ is suitable to describe the configuration $\overline{\mathrm{p}}+\mathrm{H}$ in which the electron and proton interact with each other, and the $\overline{\mathrm{p}}$ interacts with the center of mass of the electron and proton. The second one $c=2$ is suitable to describe the structure in which the electron interacts with the highly excited protonium, $\mathrm{Pn}+\mathrm{e}^{-}$. The third channel $c=3$ explicitly introduces the repulsive correlation between the antiproton and electron. The S-wave $(J=0)$ channel wavefunction having an angular momentum of $l$ on the $c$-th Jacobian coordinate is expanded in terms of Gaussian basis function with sine and cosine functions,

$$
\begin{aligned}
\psi_{c l}\left(\mathbf{r}_{c}, \mathbf{R}_{c}\right) & =\sum_{j=1}^{N_{c l}} R_{c}^{l} \exp \left(-\frac{R_{c}^{2}}{\beta_{c l, j}^{2}}\right) \sum_{i=1}^{n_{c l}}\left[A_{i j} r_{c}^{l} \exp \left(-\frac{r_{c}^{2}}{\alpha_{c l, i}^{2}}\right) \cos \left(\gamma \frac{r_{c}^{2}}{\alpha_{c l, i}^{2}}\right)\right. \\
& \left.+B_{i j} r_{c}^{l} \exp \left(-\frac{r_{c}^{2}}{\alpha_{c l, i}^{2}}\right) \sin \left(\gamma \frac{r_{c}^{2}}{\alpha_{c l, i}^{2}}\right)\right] \sum_{m}(-1)^{m} Y_{l m}\left(\hat{\mathbf{r}}_{c}\right) Y_{l m}\left(\hat{\mathbf{R}}_{c}\right) .
\end{aligned}
$$

The non-linear parameters $\alpha_{c l, i}$ and $\beta_{c l, j}$ are given according to the geometrical progression to describe both short-range correlation and long-range tail behavior. The linear coefficients $A_{i j}$ and $B_{i j}$ are determined by the variational principle for eigenenergies,

$$
\left\langle\Psi_{v 0}|H| \Psi_{v^{\prime} 0}\right\rangle=E_{v 0} \delta_{v v^{\prime}} .
$$

Since the resonance states concerned here are accosted with highly excited Pn whose number of radial nodes are more than 30, radial wave functions for $r_{c}$ should have many nodes. Thus, a fragment of the channel function $c=2$,

$$
\phi_{n l}\left(\mathbf{r}_{2}\right)=\sum_{i=1}^{n_{2 l}}\left[a_{i} r_{2}^{l} \exp \left(-\frac{r_{2}^{2}}{\alpha_{2 l, i}^{2}}\right) \cos \left(\gamma \frac{r_{2}^{2}}{\alpha_{2 l, i}^{2}}\right)+b_{i} r_{2}^{l} \exp \left(-\frac{r_{2}^{2}}{\alpha_{2 l, i}^{2}}\right) \sin \left(\gamma \frac{r_{2}^{2}}{\alpha_{2 l, i}^{2}}\right)\right] Y_{l m}\left(\hat{\mathbf{r}}_{2}\right),
$$

should have an ability to reproduce highly excited eigenstates of Pn. The Schrödinger equation for Pn is written as

$$
\left(\hat{K}_{\mathbf{r}_{2}}-\frac{1}{r_{2}}\right) \phi_{n l}=\epsilon_{n} \phi_{n l}
$$

where $\hat{K}_{\mathbf{r}_{2}}$ is a kinetic energy operator associated with $\mathbf{r}_{2}$ and $n$ is the principal quantum number of Pn. We show the $\epsilon_{n}$ convergences for Pn $(n \mathrm{~s})$ states in Fig. 2 against the number of total Gaussian basis functions $2 n_{2 l}$. One can see that the sufficient number of basis functions $2 n_{2 l} \geq 160$ provides proper eigenenergies of the highly excited $\operatorname{Pn}(n \geq 30)$.

Resonance energies and widths are determined by the help of complex scaling method [39]. A complex dilation operator is defined by

$$
U(\eta, \theta) f(\mathbf{r})=\eta^{\frac{3}{2}} e^{\frac{3}{2} i \theta} f\left(\eta e^{i \theta} \mathbf{r}\right),
$$




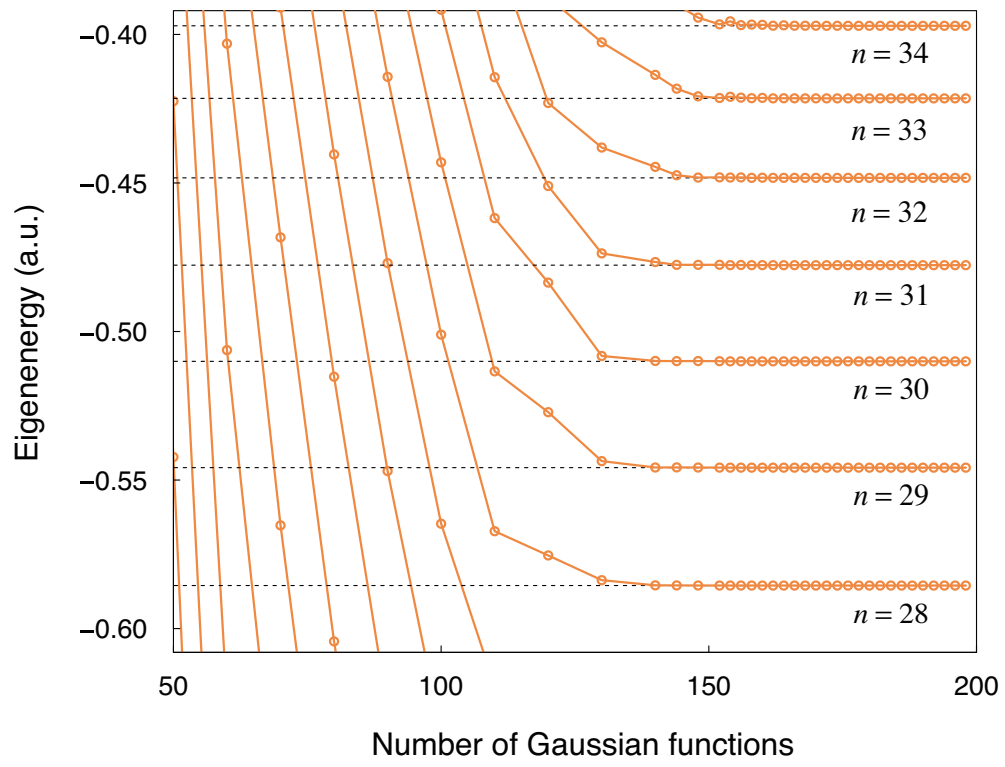

Figure 2. Eigenenergy convergences for Pn $(n s)$ in Eq. (6) are shown against the number of Gaussian functions $2 n_{2 l}$ (solid line with points). The dashed lines are the exact eigenenergies.

where $f(\mathbf{r})$ is an arbitrary function described by a coordinate $\mathbf{r}$, and $\eta$ and $\theta$ are real factors for scaling. A complex scaled Schrödinger equation is given by

$$
U(\eta, \theta) H U(\eta, \theta)^{-1} \Psi_{0}(\eta, \theta)=E(\eta, \theta) \Psi_{0}(\eta, \theta),
$$

where $E(\eta, \theta)$ is a complex eigenenergy and $\Psi_{0}(\eta, \theta)=U(\eta, \theta) \Psi_{0}$ is a complex-scaled total wavefunction. The resonance energy and width can be determined by the complex eigenvalue $E\left(\eta, \theta^{\prime}\right)=E_{\mathrm{r}}-i \Gamma / 2$ when

$$
\left.\frac{\mathrm{d} E(\eta, \theta)}{\mathrm{d} \theta}\right|_{\theta=\theta^{\prime}} \approx 0
$$

is satisfied for any $\eta$.

\section{Results and discussion}

We find two S-wave resonance states just below the $\overline{\mathrm{p}}+\mathrm{H}(1 \mathrm{~s})$ dissociation threshold. Hereafter, we distinguish the resonance states as $\mathrm{S}_{-1}$ and $\mathrm{S}_{-2}$ with energies $E_{-1}=$ -0.499728 85(4) $-i 0.00000077(2)$ and $E_{-2}=-0.499775(1)-i 0.000022(2)$, respectively, where numbers in the parentheses indicate the numerical error of the last digit.

With a trial function (noted as $\Psi_{0}^{(\mathrm{a})}$ ) consisting of three channel functions,

$$
\Psi_{0}^{(\mathrm{a})}=\psi_{10}+\psi_{11}+\psi_{20},
$$

we calculate complex eigenenergies $E(\eta, \theta)$ for $0 \leq \theta \leq 0.2$ by interval $\Delta \theta=0.01$. We find a resonance state corresponding to $\mathrm{S}_{-1}$ state just below the $\overline{\mathrm{p}}+\mathrm{H}(1 \mathrm{~s})$ threshold, $E_{\mathrm{th}}=$ -0.49972784 . Series of complex eigenenergies for resonance state $S_{-1}$ calculated with a 


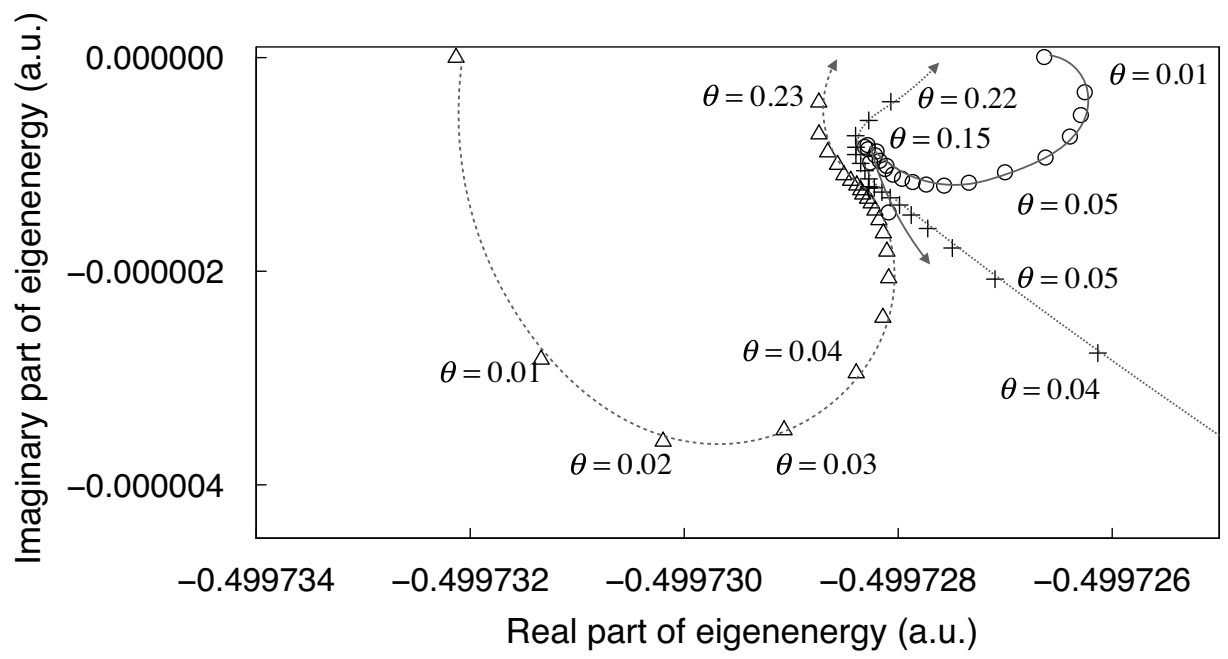

Figure 3. Complex eigenenergies $(E(\eta, \theta))$ are shown (circle: $\eta=1.0$, cross: $\eta=1.02$, triangle: $\eta=1.04)$. The regular interval of complex scaling parameter $\theta$ is $\Delta \theta=0.01$. Complex energy for resonance state $S_{-1}$ is found to be (-0.49972836(3), $0.0000017(4)$ ).

Table 1. Resonance energy and width for $S_{1}$ state calculated with trial functions consisting of different set of channel functions are shown together. The total number of basis functions is given by the sum of channel functions $N_{\text {tot }}=\sum_{c l} 2 N_{c l} n_{c l}$.

\begin{tabular}{cccc}
\hline \hline$N_{\text {tot }}$ & $\Psi$ & $E_{\mathrm{r}}$ & $\Gamma$ \\
\hline 7200 & $\psi_{10}+\psi_{20}$ & - & - \\
10800 & $\psi_{10}+\psi_{20}+\psi_{30}$ & - & - \\
10800 & $\psi_{10}+\psi_{11}+\psi_{20}$ & $-0.49972836(3)$ & $0.0000017(4)$ \\
14400 & $\psi_{10}+\psi_{11}+\psi_{20}+\psi_{30}$ & $-0.49972885(4)$ & $0.00000155(4)$ \\
\hline \hline
\end{tabular}

trial function $\Psi_{0}^{(\mathrm{a})}$ are shown in Fig. 3. Three series with different $\eta$ 's pass around a stationary point which corresponds to a complex energy of the resonance, as increasing $\theta$.

The resonance state $S_{-1}$ is examined by several different trial functions shown in Table 1 . When we construct the trial function $\Psi_{0}^{(\mathrm{b})}$ without the channel function $\psi_{11}$, namely,

$$
\Psi_{0}^{(\mathrm{b})}=\psi_{10}+\psi_{20},
$$

the resonance state is not found in the same energy region. The channel function $\psi_{10}$ gives the configuration in which the relative motion between the antiproton and the center of mass of the hydrogen atom has angular momentum $l=0$, and the $\psi_{20}$ gives the configuration in which the relative motion between the electron and the center of mass of the protonium has angular momentum $l=0$. Since the channel function $\psi_{11}$ plays a role to explicitly describe the excitation of the hydrogen atom by the electric field of the antiproton, this resonance state is expected to be supported by the induced polarization attraction. If we replace $\psi_{11}$ in the trial function $\Psi_{0}^{(\mathrm{a})}$ by $\psi_{30}$ which can introduce an explicit description between repelling 


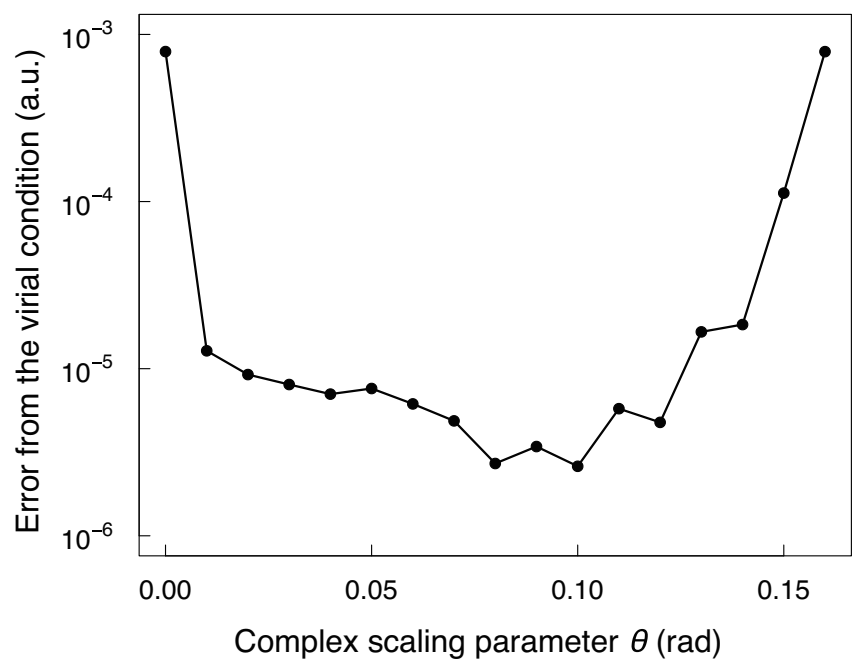

Figure 4. The deviation from the virial condition $\left|2\langle K\rangle^{(\eta, \theta)}+\langle V\rangle^{(\eta, \theta)}\right|$ (circles with a solid line) is shown against the complex scaling parameter $\theta$ for the resonance state $S_{-1}$ with the trial function in Eq. (13).

particles, antiproton and electron,

$$
\Psi_{0}^{(\mathrm{c})}=\psi_{10}+\psi_{20}+\psi_{30}
$$

gives no resonance state corresponding to the $S_{-1}$ resonance state. Therefore, the role of polarization of hydrogen atom introduced by $\psi_{11}$ is indispensable to support the resonance state. The most precise result is obtained by the four-channel trial function $\Psi_{0}^{(\mathrm{d})}$ given as

$$
\Psi_{0}^{(\mathrm{d})}=\psi_{10}+\psi_{11}+\psi_{20}+\psi_{30} .
$$

The resonance energy and width of the resonance state $\mathrm{S}_{-1}$ is determined to be $E_{\mathrm{r}}=$ $-0.49972885(4)$ and $\Gamma=0.00000155(4)$. The energy relative to the $\bar{p}+\mathrm{H}(1 \mathrm{~s})$ threshold is $E_{\mathrm{th}}-E_{\mathrm{r}}=0.00000101(4)$. Since the resonance state $\mathrm{S}_{-1}$ is located below the $\overline{\mathrm{p}}+\mathrm{H}(1 \mathrm{~s})$ threshold, the resonance width is associated with the electron detachment decay with Pn formation $\mathrm{Pn}+\mathrm{e}^{-}$. The lifetime $\tau$ is given as $\tau=\hbar / \Gamma=16 \mathrm{ps}$.

The accuracy of the resonance state $S_{-1}$ is examined by an extended virial theorem. Since the all inter-particle interactions in the Hamiltonian Eq. (1) are Coulombic interaction, the virial condition $2\langle K\rangle+\langle V\rangle=0$ is satisfied, where $K$ and $V$ are kinetic and potential energy operators, respectively. For resonance states in the complex scaling treatment, the extended virial theorem gives a similar virial condition around the resonance state [40], namely,

$$
2\langle K\rangle^{(\eta, \theta)}+\langle V\rangle^{(\eta, \theta)}=0
$$

where the symbols $\langle K\rangle^{(\eta, \theta)}$ and $\langle V\rangle^{(\eta, \theta)}$ are defined as

$$
\langle K\rangle^{(\eta, \theta)}=\int \mathrm{d} \tau \Psi(\eta, \theta) U(\eta, \theta) K \Psi(\eta, \theta),
$$


and

$$
\langle V\rangle^{(\eta, \theta)}=\int \mathrm{d} \tau \Psi(\eta, \theta) U(\eta, \theta) V \Psi(\eta, \theta)
$$

where $\mathrm{d} \tau=\mathrm{d} \mathbf{r}_{1} \mathrm{~d} \mathbf{R}_{1}=\mathrm{d} \mathbf{r}_{2} \mathrm{~d} \mathbf{R}_{2}=\mathrm{d} \mathbf{r}_{3} \mathrm{~d} \mathbf{R}_{3}$. Deviation of the $2\langle K\rangle^{(\eta, \theta)}+\langle V\rangle^{(\eta, \theta)}$ from zero can be used to estimate an accuracy of the calculated complex eigenenergy $\langle K\rangle^{(\eta, \theta)}+\langle V\rangle^{(\eta, \theta)}$. In Fig. 4, the expectation values calculated with $\Psi_{0}^{(\mathrm{d})}$ are shown against the scaling angle $\theta$. One can see that the expectation value $\left|2\langle K\rangle^{(\eta, \theta)}+\langle V\rangle^{(\eta, \theta)}\right|$ has minimum value at $\theta=0.10$ indicating the most accurate point in the series of the calculation. The relative accuracy can be estimated by $\left|2\langle K\rangle^{(\eta, \theta)}+\langle V\rangle^{(\eta, \theta)}\right| /\left|E_{-1}\right| \sim 5.9 \times 10^{-6}$.

For the further analysis of the resonance state $S_{-1}$, we compare the resonance states with the $\mathrm{BO}$ vibrational states. In the framework of the $\mathrm{BO}$ approximation, the total wavefunction of $\overline{\mathrm{pH}}$ molecule is expressed as

$$
\Psi_{0}^{\mathrm{BO}}=\phi_{\text {lep }}\left(r_{2} ; \mathbf{r}_{1}\right) \phi_{\text {nuc }}\left(r_{2}\right) Y_{00}\left(\hat{\mathbf{r}}_{1}\right)
$$

where $\mathbf{r}_{1}$ and $\mathbf{r}_{2}$ are defined by the channel $c=1$ and $c=2$. The leptonic wavefunction $\phi_{\text {lep }}\left(r_{2} ; \mathbf{r}_{1}\right)$ provides the $\mathrm{BO}$ energy $\epsilon_{\mathrm{BO}}\left(r_{2}\right)$ [23]. An effective potential for the nuclear wavefunction $\phi_{\mathrm{nuc}}\left(r_{2}\right)$ is given by using $\epsilon_{\mathrm{BO}}\left(r_{2}\right)$,

$$
V_{\mathrm{BO}}\left(r_{2}\right)= \begin{cases}-\frac{1}{r_{2}} & \left(r_{2}<R_{\mathrm{FT}}\right) \\ \epsilon_{\mathrm{BO}}\left(r_{2}\right)-\frac{1}{r_{2}} & \left(R_{\mathrm{FT}} \leq r_{2}\right)\end{cases}
$$

where $R_{\mathrm{FT}} \sim 0.639$ is the critical distance where $\epsilon_{\mathrm{BO}}\left(r_{2}\right)=0$. The asymptotic form of $V_{\mathrm{BO}}\left(r_{2}\right)$ satisfies a boundary condition $V_{\mathrm{BO}}\left(r_{2} \rightarrow \infty\right)=-\alpha_{\mathrm{d}} / 2 r_{2}^{4}+\epsilon_{\mathrm{H}}$ where $\alpha_{\mathrm{d}}=9 / 2$ is a polarizability of the hydrogen atom with infinite nuclear mass. The energies of BO states $E_{\mathrm{BO}}$ are given by the following equation

$$
\left(K_{\mathbf{r}_{2}}+V_{\mathrm{BO}}\left(r_{2}\right)\right) \phi_{v}^{\mathrm{BO}}=E_{\mathrm{BO}} \phi_{v}^{\mathrm{BO}},
$$

where $v$ is the vibrational quantum number.

The potential $V_{\mathrm{BO}}\left(r_{2}\right)$ provides a series of vibrationally excited states $(v=1-39$, BO states) [32]. We compare the resonance energy and width with the BO and recent R-matrix calculations for $\mathrm{Pn}+\mathrm{e}^{-}$scattering in Table 2. The resonance energy and the $\mathrm{BO}$ energy are given as, relative to the $\overline{\mathrm{p}}+\mathrm{H}$ threshold, $E_{\mathrm{th}}=-0.4997278$ and $E_{\mathrm{th}}^{\mathrm{BO}}=-0.5$, respectively. The highest BO state $v=39$ is located just below the dissociation threshold by 0.000048 $\mathrm{eV}$. The resonance state $\mathrm{S}_{-1}$ gives a similar but shallower energy $0.000027 \mathrm{eV}$ relative to the $\overline{\mathrm{p}}+\mathrm{H}$ threshold while the corresponding resonance state was not reported in the R-matrix calculation [32]. For the resonance state $S_{-2}$ located below the $S_{-1}$ state, complex scaling trajectories are shown in Fig. 5. The energy of the resonance state $S_{-2}$ relative to the $\bar{p}+\mathrm{H}$ threshold is $0.001274 \mathrm{eV}$ that is well consistent with the R-matrix calculation $0.001276 \mathrm{eV}$ and is shallower than the BO state $v=38$.

Since the width of the resonance state $S_{-1}$ is two orders of magnitude narrower than that of $S_{-2}$, the resonance state $S_{-1}$ is much more stable than the $S_{-2}$. Generally, for the narrow resonance states, the CSM based on the bound state approximation may be more suitable than scattering calculations like R-matrix calculation. The half of the resonance width $\Gamma / 2=0.000022 \mathrm{eV}$ for the $\mathrm{S}_{-1}$ resonance state is almost comparable to the resonance energy relative to the threshold, $0.000027 \mathrm{eV}$. Besides, the resonance width of the $\mathrm{S}_{-2}$ resonance state corresponding to $v=38$ is almost the half of the relative resonance energy by the $\overline{\mathrm{p}}+\mathrm{H}$ threshold. Therefore, the resonance state in scattering calculation near the threshold could 


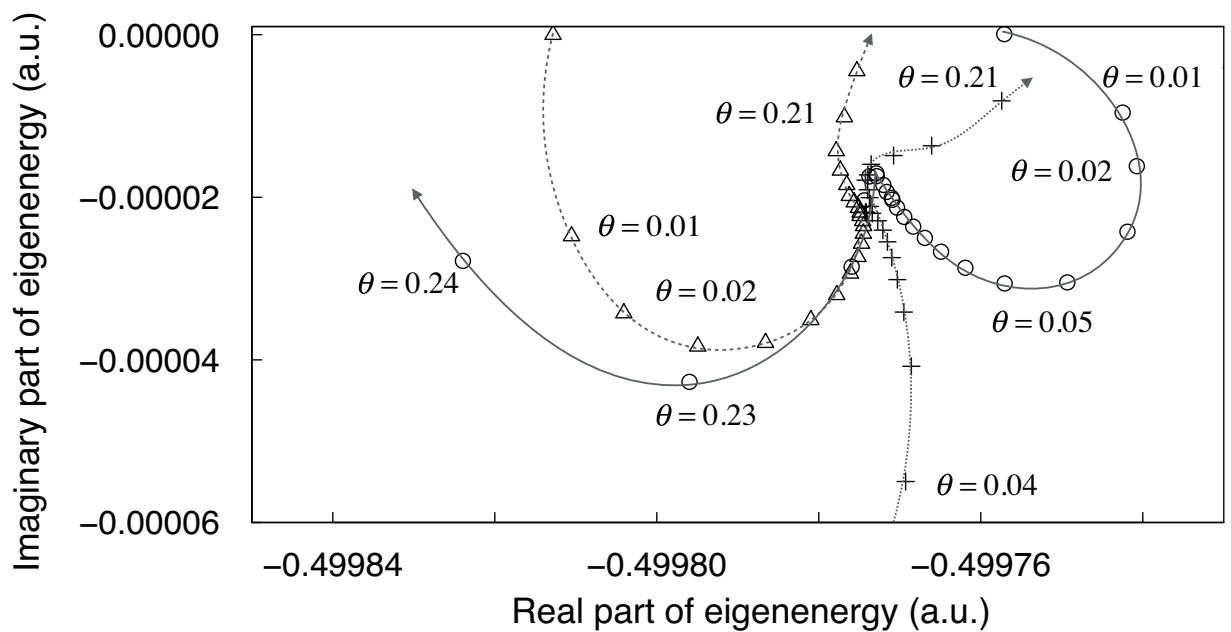

Figure 5. Complex eigenenergies $(E(\eta, \theta))$ for the $\mathrm{S}_{-2}$ resonance state are shown (circle: $\eta=1.0$, cross: $\eta=1.02$, triangle: $\eta=1.04)$. The regular interval of complex scaling parameter $\theta$ is $\Delta \theta=0.01$. The complex energy of the $\mathrm{S}_{-2}$ resonance state is found to be $(-0.499775(1),-0.000022(2))$.

Table 2. Resonance positions and widths calculated with complex scaling method (CSM), Born-oppenheimer (BO) and R-matrix calculations are shown in electron-volt $(\mathrm{eV})$. The positions are relative to the $\overline{\mathrm{p}}+\mathrm{H}$ threshold.

\begin{tabular}{lcccc}
\hline \hline & This work & \multicolumn{2}{c}{ Literature [32] } \\
\cline { 2 - 5 } & CSM & \multicolumn{2}{c}{ BO } & R-Matrix \\
\hline$E_{-1}$ & $-0.000027(0.000043)$ & $v=39$ & -0.000048 & - \\
$E_{-2}$ & $-0.001274(0.001175)$ & $v=38$ & -0.001616 & $-0.001276(0.00105)$ \\
\hline \hline
\end{tabular}

be influenced by the existence of the open dissociation channel $\bar{p}+\mathrm{H}$, and also by the $\mathrm{S}_{-2}$ resonance tail.

For the resonance states $\mathrm{S}_{-1}$ and $\mathrm{S}_{-2}$, one can see that the non-adiabatic calculation gives shallower energy than the BO calculation, which implies that the adiabatic picture cannot be adoptable quantitatively to the interpretation of the compound $\overline{\mathrm{p}} \mathrm{H}$.

\section{Conclusion}

We non-adiabatically analyze the resonance states just below the $\overline{\mathrm{p}}+\mathrm{H}$ dissociation threshold with GEM and CSM. The total wavefunction is constructed by several channel functions defined in rearrangement Jacobian coordinates that describes both major particle correlations $\overline{\mathrm{p}}+\mathrm{H}$ and $\mathrm{Pn}+\mathrm{e}^{-}$explicitly. The highly excited states of $\mathrm{Pn}$ in the dissociation states are reproduced by a number of oscillation Gaussian functions.

We find two resonance states $S_{-1}$ and $S_{-2}$ below the $\bar{p}+\mathrm{H}(1 \mathrm{~s})$ threshold. The energy and width of the lower resonance state $S_{-2}$ are in good agreement with the recent R-matrix calculation [32], and the other resonance state $S_{-1}$ is newly found. The resonance state $S_{-1}$ 
located $0.000027 \mathrm{eV}$ below the $\overline{\mathrm{p}}+\mathrm{H}(1 \mathrm{~s})$ threshold is examined by several trial wavefunctions. The channel function having a role to introduce a polarization effect of hydrogen atom is indispensable to support the resonance state. It suggests that the resonance state is caused by the induced polarization potential of the hydrogen atom by the antiproton. The accuracy of the present calculation is evaluated by the extended virial theorem.

The resonance states $\mathrm{S}_{-1}$ and $\mathrm{S}_{-2}$ are located near the $v=39$ and $v=38$ BO states, respectively. However, since relative energies to the $\bar{p}+\mathrm{H}(1 \mathrm{~s})$ threshold are almost the same values as the widths, the resonance states should affect the scattering cross section above the threshold. This effect should be significant for experiments using ultra low energy $\bar{p}$ because the cross section of $\overline{\mathrm{p}}$ and residual hydrogen atoms may increase. In the BO potential given in Eq. (18), the contribution from the electron is omitted inside the critical distance. The non-adiabatic calculation, which can take the contribution of the electron inside the critical distance to the total energy into account and can include the electron detachment decay associated with the Pn formation, improves the energy relative to the $\overline{\mathrm{p}}+\mathrm{H}(1 \mathrm{~s})$ threshold by 0.000021 a.u. (equivalently $6.6 \mathrm{~K}$ ) for $v=39 \mathrm{BO}$ state and 0.000342 a.u. (108 K) for $v=38$ BO state.

\section{Acknowledgment}

This work was financially supported by a Grant-in-Aid for Scientific Research from the Ministry of Education, Culture, Sports, Science and Technology (MEXT), Japan, a Kakenhi Grant-in-Aid from the Japan Society for the Promotion of Science (JSPS), and by the Division for Interdisciplinary Advanced Research and Education (DIARE), Tohoku University. The computation was conducted on the supercomputers at Kyushu University.

\section{References}

[1] A. Ermolaev, Phys. Lett. A 149, 151 (1990)

[2] N. Toshima, Phys. Lett. A 175, 133 (1993)

[3] J. Azuma, N. Toshima, K. Hino, A. Igarashi, Phys. Rev. A 64, 062704 (2001)

[4] A.B. Voitkiv, J. Ullrich, Phys. Rev. A 67, 062703 (2003)

[5] K. Sakimoto, Physica Scripta 2004, 271 (2004)

[6] T. Walcher, Ann. Rev. Nucl. Part. Sci. 38, 67 (1988)

[7] C. Amsler, F. Myhrer, Ann. Rev. Nucl. Part. Sci. 41, 219 (1991)

[8] H. Knudsen, U. Mikkelsen, K. Paludan, K. Kirsebom, S.P. Møller, E. Uggerhøj, J. Slevin, M. Charlton, E. Morenzoni, Phys. Rev. Lett. 74, 4627 (1995)

[9] S. Ulmer, C. Smorra, A. Mooser, K. Franke, H. Nagahama, G. Schneider, T. Higuchi, S.V. Gorp, K. Blaum, Y. Matsuda et al., Nature 524, 196 (2005)

[10] M. Ahmadi, B.X.R. Alves, C.J. Baker, W. Bertsche, E. Butler, A. Capra, C. Carruth, C.L. Cesar, M. Charlton, S. Cohen et al., Nature 541, 506 (2016)

[11] M. Ahmadi, B.X.R. Alves, C.J. Baker, W. Bertsche, E. Butler, A. Capra, C. Carruth, C.L. Cesar, M. Charlton, S. Cohen et al., Nature 548, 66 (2017)

[12] M. Diermaier, C.B. Jepsen, B. Kolbinger, C. Malbrunot, O. Massiczek, C. Sauerzopf, M.C. Simon, J. Zmeskal, E. Widmann, Nat. Commun. 8, 15749 (2017)

[13] H. Nagahama, C. Smorra, S. Sellner, J. Harrington, T. Higuchi, M. Borchert, T. Tanaka, M. Besirli, A. Mooser, G. Schneider et al., Nat. Commun. 8, 14084 (2017)

[14] C. Smorra, A. Mooser, M. Besirli, M. Bohman, M. Borchert, J. Harrington, T. Higuchi, H. Nagahama, G. Schneider, S. Sellner et al., Phys. Lett. B 769, 1 (2017) 
[15] M. Amoretti, C. Amsler, G. Bonomi, A. Bouchta, P. Bowe, C. Carraro, C.L. Cesar, M. Charlton, M.J.T. Collier, M. Doser et al., Nature 419, 456 (2002)

[16] T.A. Collaboration, Nat. Phys. 7, 558 (2011)

[17] C. Smorra, K. Blaum, K. Franke, Y. Matsuda, A. Mooser, H. Nagahama, C. Ospelkaus, W. Quint, G. Schneider, S. Van Gorp et al., Hyperfine Interact. 228, 31 (2014)

[18] C. Smorra, A. Mooser, K. Franke, H. Nagahama, G. Schneider, T. Higuchi, S. Gorp, K. Blaum, Y. Matsuda, W. Quint et al., Int. J. Mass Spectrom. 389, 10 (2015)

[19] C. Smorra, K. Blaum, L. Bojtar, M. Borchert, K. Franke, T. Higuchi, N. Leefer, H. Nagahama, Y. Matsuda, A. Mooser et al., Eur. Phys. J. Special Topics 224, 3055 (2015)

[20] B. Zygelman, A. Saenz, P. Froelich, S. Jonsell, A. Dalgarno, Phys. Rev. A 63, 052722 (2001)

[21] P. Berggren, H. Stegeby, A. Voronin, P. Froelich, J. Phys. B: At. Mol. Opt. Phys. 41, $155202(2008)$

[22] H. Stegeby, K. Piszczatowski, H. Karlsson, R. Lindh, P. Froelich, Cent. Eur. J. Phys. 10, 1038 (2012)

[23] J.E. Turner, V.E. Anderson, K. Fox, Phys. Rev. 174, 81 (1968)

[24] J.E. Turner, Am. J. Phys. 45, 758 (1977)

[25] E.A.G.Armour, J.M.Carr, V.Zeman, J. Phys. B: At. Mol. Opt. Phys. 31, L679 (1998)

[26] K. Strasburger, J. Phys. B: At. Mol. Opt. Phys. 37, 4483 (2004)

[27] A. Voronin, J. Carbonell, Hyperfine Interact. 115, 143 (1998)

[28] P. Froelich, S. Jonsell, A. Saenz, B. Zygelman, A. Dalgarno, Phys. Rev. Lett. 84, 4577 (2000)

[29] B. Zygelman, A. Saenz, P. Froelich, S. Jonsell, Phys. Rev. A 69, 042715 (2004)

[30] A.Y. Voronin, P. Froelich, Phys. Rev. A 77, 022505 (2008)

[31] E. Garrido, D. Fedorov, A. Jensen, Phys. Lett. B 684, 132 (2010)

[32] K. Sakimoto, Phys. Rev. A 90, 032514 (2014)

[33] H. Stegeby, K. Piszczatowski, J. Phys. B: At. Mol. Opt. Phys. 49, 014002 (2016)

[34] E. Hiyama, Y. Kino, M. Kamimura, Prog. Part. Nucl. Phys. 51, 223 (2003)

[35] T. Yamashita, Y. Kino, Eur. Phys. J. D 70, 1 (2016)

[36] M. Umair, S. Jonsell, J. Phys. B: At. Mol. Opt. Phys. 50, 044001 (2017)

[37] T. Yamashita, M. Umair, Y. Kino, J. Phys. B: At. Mol. Opt. Phys. 50, 205002 (2017)

[38] K. Piszczatowski, A. Voronin, P. Froelich, Phys. Rev. A 89, 062703 (2014)

[39] Y.K. Ho, Phys. Rep. 99, 1 (1983)

[40] E. Brändas, P. Froelich, Phys. Rev. A 16, 2207 (1977) 